of ENM-bio-interactions over the entire life span. Age-resolved analysis identified especially middle-aged worms as vulnerable target group to certain ENMs. In vulnerable groups, the distribution of ENMs throughout the cytoplasm and the cell nucleus in single intestinal and vulval cells as well as in the pharyngeal tissue was correlated with functional alterations. Nano silica induces nuclear amyloid and perturbs the peptide metabolism in intestinal cells. Global protein is reduced which promotes a 'petite' phenotype. Consistently, mass spectrometry analysis identifies a silica NP-induced aggregome network that contains predominantly the gene ontology (GO) groups of proteostasis, metabolic processes, rRNA processing and translation. Among the molecular ENM-effects, nano silica-exposed worms show an early onset of age-associated stigmata including widespread protein aggregation and premature neurodegeneration of serotonergic and dopaminergic neurons. Impaired neurosignaling is related with reproductive and locomotion defects. All age-related defects result in reduced fitness and health span which normally occur in old worms but are prematurely induced by certain ENMs like nano silica. System biology-based analyses have the potential to identify common pathways of nanoparticle-bio-interactions across species from worm via other taxa to human and thus, to evaluate the risk of nano silica to human health.

References: A Scharf, A Piechulek, A von Mikecz, ACS Nano 7, 12 (2013) A Scharf, KH Gührs, A von Mikecz, Nanotoxicology 10, 4 (2016) A Piechulek, A von Mikecz, Environ Pollut 233 (2018) A von Mikecz, Environ Sci: Nano 5 (2018) doi: http://dx.doi.org/10.7124/bc.0009CC

\section{The cell nucleus as sensor of environmental pollution: Amyloid, neurodegeneration and aging}

Aneta Piechulek ${ }^{1}$, Lutz Berwanger ${ }^{1}$, Andrea Scharf $^{2}$, and Anna von Mikecz ${ }^{1}$

${ }^{1}$ IUF - Leibniz Research Institute of Environmental Medicine at Heinrich-Heine-University Duesseldorf, Duesseldorf, Germany; ${ }^{2}$ Washington University, St. Louis, USA

mikecz@tec-source.de

Two major environmental challenges are predominant - climate change and the biodiversity crisis. Also, pollutants are distributed globally. We identified the cell nucleus as a sensitive sensor for bio-effects of pollutants such as mercury and nanoparticles. Our investigations focus on the role of pollutant-induced nuclear amyloid formation in neural signaling, neurotoxicity and accelerated aging in the nematode Caenorhabditis elegans. Methods: At present, the majority of investigations on the toxicology of pollutants address short-term effects. While this approach allows for the identification of uptake pathways, exposition and acute toxicity, xenobiotic-organism interactions that manifest later in an adult life are missed. To characterize effects of pollutants over the entire life span, all analyses are performed in the nematode C. elegans. Results: We show that mercury and nano silica effect widespread protein aggregation. Proteomic profiling revealed that both pollutants promote segregation of proteins belonging to the gene ontology (GO) group of 'protein folding, proteolysis and stress response' to an SDS-resistant aggregome network. Candidate proteins in this 
group include chaperones, heat shock proteins and subunits of the $26 \mathrm{~S}$ proteasome which are all decisively involved in protein homeostasis. Another emerging GO group is translation. Crucial proteins such as the methyltransferase fibrillarin aggregate, segregate and thus reduce translational activity. The metabolic changes contribute to neurodegeneration and starvation which in turn manifests in petite worms showing motoric and olfactory deficits. Conclusions: A picture emerges that pollutants induce premature aging, thereby reducing the health span and longevity of $\mathrm{C}$. elegans. The neural system of the worm represents a prominent target organ showing neuromuscular defects that among others manifest in neurodegeneration of single neurons in combination with altered locomotion, reduced fecundity and impaired olfaction. As C. elegans leads a dual life, e.g. in the lab and in ecosystems, our results explain how environmental pollutants reduce the fitness of wild worms. Concerning the survival of wild populations the flexibility of adaptive gene expression and proteomes likely determines biodiversity, namely resilience against pollutantand climate-stress.

doi: http://dx.doi.org/10.7124/bc.0009CD

\section{Non-random chromosome segregation in Mesostoma ehrenbergii spermatocytes}

Eleni Fegaras and Arthur Forer

Biology Department, York University, Toronto, ON Canada M3J 1P3

efegaras@gmail.com
Mesostoma erhenbergii are hermaphroditic aquatic flatworms that reside in rivers and lakes across North America and elsewhere. Their primary spermatocytes have a very unusual meiosis (reviewed by Ferraro-Gideon et al. 2014). The cells have four univalent chromosomes, of two different kinds, that stay at the spindle poles, plus three bivalents. Some evidence suggests that the chromosomes are distributed via non-random segregation. For example, univalents move from pole to pole to obtain proper segregation, but they do this more often than necessary to obtain one of each kind at each pole, and often homologous chromosomes switch places with one another (Oakley 1984). Another example is that oriented bivalents sometimes detach and reorient to the opposite poles (Ferraro-Gideon et al 2014). Here we provide further evidence of non-random segregation in Mesostoma spermatocytes. Methods: Testes extracted from living Mesostoma ehrenbergii using a pulled glass needle are expelled onto a coverslip into Ringers solution that contained fibrinogen, and are held in a fibrin clot. Nocodazole (NOC) was perfused during prometaphase. We recorded live cells and studied the time-lapsed images. Results: NOC depolymerizes spindle microtubules. According to standard theories of mitosis, if microtubules are quickly depolymerized the chromosomes should arrest at the metaphase plate and not move. In Mesostoma spermatocytes, however, immediately following drug addition the chromosomes stretch out and, after a few minutes, in 52/59 cells the bivalent kinetochores detach from one pole and all move rapidly toward the other pole (Fegaras and Forer 2018 a and b). We have eliminated some other possibilities, and sug- 